\title{
Sharp-interface projection of a fluctuating phase-field model
}

\author{
R. Benítez and L. Ramírez-Piscina \\ Departament de Física Aplicada, \\ Universitat Politècnica de Catalunya, \\ Doctor Marañón 44, \\ E-08028 Barcelona, Spain.
}

(Dated: November 14, 2018)

\begin{abstract}
We present a derivation of the sharp-interface limit of a generic fluctuating phase-field model for solidification. As a main result, we obtain a sharp-interface projection which presents noise terms in both the diffusion equation and in the moving boundary conditions. The presented procedure does not rely on the fluctuation-dissipation theorem, and can therefore be applied to account for both internal and external fluctuations in either variational or non-variational phasefield formulations. In particular, it can be used to introduce thermodynamical fluctuations in non-variational formulations of the phase-field model, which permit to reach better computational efficiency and provide more flexibility for describing some features of specific physical situations. This opens the possibility of performing quantitative phase-field simulations in crystal growth while accounting for the proper fluctuations of the system.

PACS numbers: 81.10.Aj,81.30.Fb,05.40.-a,68.08.-p,64.70.Dv
\end{abstract}




\section{INTRODUCTION}

In recent years, phase-field models have emerged as an efficient technique to simulate interfacial phenomena in non-equilibrium systems [1]. This method has mainly been developed for solidification [2, 3, 4], but has also successfully been applied to other problems, such as grain boundaries [5], crack propagation [6], viscous fingering [7] or vesicle dynamics 8]. The phase-field approach introduces an equation for a continuous variable $\phi(\mathbf{r}, t)$, which appears as an order parameter, and takes distinct, constant values in the different phases. The interface is then described by the level set $\phi=$ constant, and the transition between both phases takes place in a diffuse interface of thickness $W$. The model is completed by coupling the $\phi$ equation with a diffusion field which acts as a driving force for the motion of the front. The behavior of the diffuse interface can then be computed by the integration of a set of partial differential equations for the whole system, therefore avoiding the explicit tracking of the interface position. This has practical advantages over using the free boundary conditions that are characteristic of a moving boundary description. Phase-field models are usually constructed to recover the classical moving boundary dynamics in the so called sharp-interface limit as $W \rightarrow 0$ [9]. This limit is taken by means of a systematic asymptotic expansion on the interface width, and allows the model parameters to be determined in terms of the physical properties of the system.

In early phase-field formulations, the model equations were derived from the variational minimization of a global free-energy functional for the heterogeneous system. Such variational formulations, however, in spite of their appealing structure, presented poor computational efficiency and did not permit to obtain truly quantitative results. For this reason, recently proposed phase-field models are not derived from a variational principle, but are specifically constructed to recover a certain moving boundary problem in the sharp-interface limit [7, 10]. Besides presenting a better computational behavior, non-variational phase-field formulations provide for more flexibility in the description of some particular features such as different transport properties in the solid and liquid phases [11, 12].

On the other hand, the presence of fluctuations has always been an important issue in the study of pattern-forming instabilities in crystal growth [13, 14]. Indeed, internal or external noises play the role of an initiation mechanism for the morphological deformations of the interface [15, 16]. Thermal or solute fluctuations, for instance, must be taken into account 
in order to study important problems such as the dynamical selection of the primary spacing in directional solidification [16] or the formation of secondary instabilities (sidebranches) in dendritic growth [17]. Fluctuations were soon introduced into phase-field models in an ad hoc way as a controlled source of interfacial perturbations [18]. However, phase field models accounting for internal thermodynamical fluctuations have not been proposed until recently, and in the context of variational formulations [19, 20, 21, 22]. In such variational cases, the statistical properties of the fluctuating terms can straightforwardly be determined by using the fluctuation-dissipation theorem, following the lines applied by Hohenberg and Halperin within the context of critical dynamics [23]. In non-variational formulations, however, the fluctuation-dissipation relation becomes useless for this purpose because the dynamics of the system cannot be derived from a single free-energy functional.

The aim of this work is to present a systematic procedure to account for the introduction of generic sources of noise in either variational or non-variational phase-field models. To this end, we will perform the sharp-interface limit of a fluctuating phase-field model for solidification and explicitely obtain the properties of the projected noise terms that will appear in the moving boundary equations. This projection, which does not rely on the fluctuationdissipation theorem, will be carried out by means of a hybrid asymptotic expansion which combines a standard sharp-interface limit with a small noise assumption for the intensities of the noise terms in the model. The structure of the resulting sharp-interface projection takes the form of a moving boundary problem, which now includes bulk and interfacial stochastic terms. The statistical properties of these new terms are related to those of the noises appearing in the starting phase-field equations. The extension of our procedure to thin-interface asymptotics [4] is straightforward and is not presented here for the sake of clarity.

As a particular case, this analytical technique will enable a prescription for the introduction of internal thermodynamical fluctuations in non-variational phase-field models, subject only to the constraint of providing the correct interface equilibrium fluctuations. This approach will also allow for the consideration of more general noise sources of an external origin, such as experimental imperfections or controlled perturbations, which do not follow equilibrium statistics. It is worth pointing out that while the calculations will be performed within the framework of the symmetric solidification model, the approach can be easily extended to one-sided formulations [12]. 
This work has been organized as follows: The stochastic model equations are presented in Sec. II. The asymptotic stochastic procedure is developed in Sec. III which has been divided in four different subsections: Sec. ПIA and ЩВ are dedicated to find solutions of the equations in the inner and outer asymptotic regions, respectively. The solvability conditions for the inner expansion are imposed in Sec. IIC, whereas in Sec. IIID we perform the asymptotic matching between the inner and outer stochastic fields in order to obtain the form of the projected equations. The projected problem is then compared in Sec. IV with the standard Lanvegin formulation for solidification [13, 14], allowing for the determination of the model parameters in the case of having internal noises of a thermodynamical origin. A numerical test for the validity of the approach is reported in Sec. V] and Sec. VI is devoted to present some discussion and concluding remarks.

\section{MODEL EQUATIONS}

Our approach starts from a generic non-variational phase-field model, which applies for both the solidification of a pure substance and for the symmetric solidification of a dilute alloy with a constant miscibility gap [4],

$$
\begin{aligned}
\alpha \varepsilon^{2} \partial_{t} \phi & =\varepsilon^{2} \nabla^{2} \phi-f^{\prime}(\phi)-\varepsilon \lambda g^{\prime}(\phi) u+\varepsilon^{\frac{3}{2}} \eta(\mathbf{r}, t) \\
\partial_{t} u & =\nabla^{2} u+\frac{1}{2} \partial_{t} h(\phi)-\nabla \cdot \mathbf{q}(\mathbf{r}, t)
\end{aligned}
$$

where $\alpha$ is a parameter determining the time scale of the phase-field dynamics and $\lambda$ accounts for the coupling strength between $\phi$ and the diffusion field $u$. We choose $g(\phi)$ and $h(\phi)$ to be odd polynomial functions of $\phi$ satisfying the limiting conditions $g^{\prime}( \pm 1)=0$ and $h( \pm 1)= \pm 1$, and $f(\phi)$ to be given by the standard double-well potential

$$
f(\phi)=\frac{1}{4} \phi^{4}-\frac{1}{2} \phi^{2}
$$

In the model equations Eqs. (2.1), (2.2), $u$ is a reduced diffusive field defined by $u=$ $\left(T-T_{M}\right) /(L / c)$ in the case of pure substances and by $u=\left(c-\frac{1}{2}\left(c_{S}^{0}+c_{L}^{0}\right)\right) / \Delta c_{0}+\frac{1}{2} g(\phi)$ for symmetric alloys, where $T_{M}$ is the melting temperature, $L$ the latent heat per unit volume, $c$ the specific heat per unit volume and $\Delta c_{0} \equiv c_{L}^{0}-c_{S}^{0}$, being $c_{S}^{0}, c_{L}^{0}$ the solid and liquid equilibrium concentrations of the alloy, respectively. The two minima $\phi= \pm 1$ of $f(\phi)$ in Eq. (2.3) correspond respectively to the solid and liquid phases of the system, so the 
interface will be represented by the transition zone between these two values. Space and times in Eqs. (2.1), (2.2) have been scaled out using a characteristic length $l$ and a time scale $\nu=l^{2} / D$, where $D$ is the thermal or chemical diffusivity of the substance. The control parameter $\varepsilon=W / l$ is the scaled interface thickness, and will be the small parameter in which the formal expansions will be carried out.

Fluctuations appear in the model as a non-conserved noise term $\eta$ in the equation for the phase-field, and as a conserved stochastic current $\mathbf{q}$ in the diffusion equation. These fluctuations account for generic noise sources of either an internal or an external origin. We assume that the noises are white and Gaussian with correlations given by

$$
\begin{aligned}
\left\langle\eta(\mathbf{r}, t) \eta\left(\mathbf{r}^{\prime}, t^{\prime}\right)\right\rangle & =2 \sigma_{\phi}^{2} \delta\left(\mathbf{r}-\mathbf{r}^{\prime}\right) \delta\left(t-t^{\prime}\right) \\
\left\langle q_{i}(\mathbf{r}, t) q_{j}\left(\mathbf{r}^{\prime}, t^{\prime}\right)\right\rangle & =2 \sigma_{u}^{2} \delta_{i j} \delta\left(\mathbf{r}-\mathbf{r}^{\prime}\right) \delta\left(t-t^{\prime}\right)
\end{aligned}
$$

In the proposed phase field model, parameters such as $\alpha, \lambda$, and the noise amplitudes $\sigma_{\phi}$, $\sigma_{u}$, are intended to represent (or to be directly related to) physical parameters. On the contrary, the scaled interface width $\varepsilon$ has been introduced as an expansion parameter. As a matter of fact, Eqs. (2.1), (2.2) have been constructed so that the resulting dynamics (in the double limit of sharp interface and small noise) will be independent of $\varepsilon$. In particular, the scaling factor $\epsilon^{3 / 2}$ of the the noise term in Eq. (2.1) has been introduced in order to make the fluctuations of the interfacial dynamics, as will be obtained below, independent of $\epsilon$. The details of this calculation and the presentation of the results are given in the next section.

\section{HYBRID ASYMPTOTIC EXPANSION}

In order to deal with fluctuating phase-field models, the standard asymptotic expansion, performed in terms of a small interface thickness, should be complemented with a small noise assumption. The combination of these approaches will give rise to a hybrid asymptotic procedure. To this end, the small noise assumption will be imposed by assuming that $\sigma_{\phi}, \sigma_{u}$ obey some order relations with the interface thickness $\varepsilon$. Namely, we take

$$
\begin{aligned}
\sigma_{\phi} & \sim O\left(\varepsilon^{3 / 2}\right), \\
\sigma_{u} & \sim O\left(\varepsilon^{2}\right),
\end{aligned}
$$


which will permit along the expansion procedure to maintain the fluctuating terms as small perturbations at the desired order in a consistent way. Relations (3.1), (3.2) should not be understood as any explicit dependence of these parameters on $\varepsilon$, but only as a way to formalize a double expansion in terms of a single vanishing parameter, the interface thickness, $\varepsilon \rightarrow 0$.

Our method closely follows the standard asymptotic procedure described in Ref. [1]]. We start by dividing the system into two different regions: an outer region far from the interface at distances much greater than $\varepsilon$, where the phase field presents the two constant values $\phi= \pm 1$ representing the solid and liquid phases at each side of the interface, and an inner region located around the interface up to distances of order $\varepsilon$, where the phase field varies between these two values. In the limit $\varepsilon \rightarrow 0$, solutions for the fields in both regions should match order by order in $\varepsilon$ at some intermediate distance $r_{M}$, which can be taken of order $r_{M} \sim \varepsilon^{1 / 2}$.

\section{A. Outer region}

In the outer region, the equations can be solved at each order by expanding the fields in powers of $\varepsilon$ as

$$
\begin{aligned}
& u=u_{0}+\varepsilon u_{1}+\varepsilon^{2} u_{2}+O\left(\varepsilon^{3}\right), \\
& \phi=\phi_{0}+\varepsilon \phi_{1}+\varepsilon^{2} \phi_{2}+O\left(\varepsilon^{3}\right),
\end{aligned}
$$

and by expanding in Taylor series around $\phi=\phi_{0}$ the functions $f, g$ appearing in the model equations. If we use the order relations Eqs. (3.1), (3.2), the noise terms can be assumed to be of orders

$$
\begin{aligned}
& \eta \sim O\left(\varepsilon^{3 / 2}\right) \\
& \mathbf{q} \sim O\left(\varepsilon^{2}\right)
\end{aligned}
$$

and the outer equations can then be obtained at each order in $\varepsilon$. 


\section{Zero Order}

At the leading order $\left(\varepsilon^{0}\right)$, the outer equations are given by

$$
\begin{aligned}
f^{\prime}\left(\phi_{0}\right) & =0 \\
\partial_{t} u_{0} & =\nabla^{2} u_{0}+\frac{1}{2} \partial_{t} h\left(\phi_{0}\right) .
\end{aligned}
$$

Introducing the function $f(\phi)$ into Eq. (3.7), we obtain $\phi_{0}= \pm 1$, and using that $h( \pm 1)= \pm 1$, Eq. (3.8) adopts the form

$$
\partial_{t} u_{0}=\nabla^{2} u_{0}
$$

\section{First Order}

At first order in $\varepsilon$, we find

$$
\begin{aligned}
f^{\prime \prime}\left(\phi_{0}\right) \phi_{1} & =-\lambda g^{\prime}\left(\phi_{0}\right) u_{0} \\
\partial_{t} u_{1} & =\nabla^{2} u_{1}+\frac{1}{2} \partial_{t}\left[\phi_{1} h\left(\phi_{0}\right)\right] .
\end{aligned}
$$

From Eq. (3.10) we determine $\phi_{1}=0$ by noting that the functions $f, g$ satisfy $f^{\prime \prime}( \pm 1) \neq 0$ and $g^{\prime}( \pm 1)=0$, and introducing $\phi_{1}=0$ into Eq. (3.11) we get

$$
\partial_{t} u_{1}=\nabla^{2} u_{1}
$$

\section{Second Order}

At second order $\left(\varepsilon^{2}\right)$, and using that $\phi_{1}=0$, the random current $\mathbf{q}$ appears in the equation for the outer diffusive field

$$
\begin{aligned}
f^{\prime \prime}\left(\phi_{0}\right) \phi_{2} & =-\lambda g^{\prime}\left(\phi_{0}\right) u_{1} \\
\partial_{t} u_{2} & =\nabla^{2} u_{2}+\frac{1}{2} \partial_{t}\left[h^{\prime}\left(\phi_{0}\right) \phi_{2}\right]-\nabla \cdot \mathbf{q} .
\end{aligned}
$$

Using that $g^{\prime}( \pm 1)=0$ and $f^{\prime \prime}( \pm 1) \neq 0$, equation (3.13) is solved by $\phi_{2}=0$, and the second term at the right hand side of Eq. (3.14) can be neglected. Collecting the results obtained at the three first orders, the outer fields are given, up to second order in $\varepsilon$, by

$$
\begin{aligned}
\phi & = \pm 1+O\left(\varepsilon^{3}\right), \\
\partial_{t} u & =\nabla^{2} u-\nabla \cdot \mathbf{q}(\mathbf{r}, t)+O\left(\varepsilon^{3}\right) .
\end{aligned}
$$




\section{B. Inner region}

For the inner region, we write Eqs. (2.1), (2.2) in a curvilinear coordinate system centered at the interface. The idea is that the solvability condition for the very existence of solutions of these transformed equations will provide the evolution of the coordinate system, i.e. of the interface, which in fact constitutes the solution we are looking for. To define this coordinate system by maintaining it smooth at small scales, we use an auxiliary coarse grained field defined as a local spatial and temporal average of the fluctuating field $\phi$. The surface corresponding to the level set of this coarse grained field $\langle\phi(\mathbf{r}, t)\rangle=0$ allows to define the $3 \mathrm{D}$ orthogonal curvilinear coordinate system $\left(r, s_{1}, s_{2}\right)$, where $r$ is a normal distance from the surface and $s_{1}, s_{2}$ are the arclength distances measured along the principal curvature directions of the surface. Furthermore, we introduce in the inner region the scaled normal coordinate $\rho=r / \varepsilon$ and the scaled time $\tau=t / \varepsilon$. We use capital letters to refer to all the fields when written in the inner region. After some manipulation, and keeping terms up to second order in $\varepsilon$, we obtain the inner equations in the frame of the moving interface

$$
\begin{gathered}
\alpha \varepsilon\left[\frac{d}{d \tau}-v \partial_{\rho}\right] \Phi=\partial_{\rho}^{2} \Phi+\varepsilon \kappa \partial_{\rho} \Phi-\varepsilon^{2} \rho\left(\kappa^{2}-2 \Pi\right) \partial_{\rho} \Phi \\
+\varepsilon^{2} \sum_{i=1,2} \partial_{s_{i}}^{2} \Phi-f^{\prime}(\Phi)-\varepsilon \lambda g^{\prime}(\Phi) U+\varepsilon^{1 / 2} H(\rho, \mathbf{s}, \tau), \\
\frac{1}{\varepsilon}\left[\frac{d}{d \tau}-v \partial_{\rho}\right] U=\frac{1}{\varepsilon^{2}} \partial_{\rho}^{2} U+\frac{1}{\varepsilon}\left[\kappa-\varepsilon \rho\left(\kappa^{2}-2 \Pi\right)\right] \partial_{\rho} U \\
+\sum_{i=1,2} \partial_{s_{i}}^{2} U-\frac{v}{2 \varepsilon} \partial_{\rho} h(\Phi)+\frac{1}{2 \varepsilon} \frac{d h(\Phi)}{d \tau}-\frac{1}{\varepsilon^{2}} \partial_{\rho} Q_{\rho}(\rho, \mathbf{s}, \tau),
\end{gathered}
$$

where $v=v(\mathbf{s}, \tau)$ is the local normal velocity of the interface, and we have introduced $\kappa=\kappa_{1}+\kappa_{2}$ and $\Pi=\kappa_{1} \kappa_{2}$ as the mean and Gaussian curvatures of the surface, being $\kappa_{1}(\mathbf{s}, \tau), \kappa_{2}(\mathbf{s}, \tau)$ its principal curvatures.

The fluctuating functions $H(\rho, \mathbf{s}, \tau)=\varepsilon \eta(\mathbf{r}, t)$ and $\mathbf{Q}(\rho, \mathbf{s}, \tau)=\varepsilon \mathbf{q}(\mathbf{r}, t)$ in Eqs. (3.17), (3.18) stand for the renormalized noises in the inner region, and $Q_{\rho}$ corresponds to the normal component of the stochastic current $\mathbf{Q}$. The correlations of these noise terms are given by

$$
\begin{aligned}
\left\langle H(\rho, \mathbf{s}, \tau) H\left(\rho^{\prime}, \mathbf{s}^{\prime}, \tau^{\prime}\right)\right\rangle & =2 \sigma_{\phi}^{2} \delta\left(\rho-\rho^{\prime}\right) \delta\left(\mathbf{s}-\mathbf{s}^{\prime}\right) \delta\left(\tau-\tau^{\prime}\right), \\
\left\langle Q_{i}(\rho, \mathbf{s}, \tau) Q_{j}\left(\rho^{\prime}, \mathbf{s}^{\prime}, \tau^{\prime}\right)\right\rangle & =2 \sigma_{u}^{2} \delta_{i j} \delta\left(\rho-\rho^{\prime}\right) \delta\left(\mathbf{s}-\mathbf{s}^{\prime}\right) \delta\left(\tau-\tau^{\prime}\right),
\end{aligned}
$$


so that the orders in $\varepsilon$ of $H(\rho, \mathbf{s}, \tau)$ and $\mathbf{Q}(\rho, \mathbf{s}, \tau)$ are those of $\sigma_{\phi}$ and $\sigma_{u}$ respectively. Note that the renormalization of the noise terms is a direct consequence of the scaling of the $t, r$ coordinates in the inner region. Indeed, noise terms give rise to an $\epsilon$ factor when written in the inner region due to the rescaling in both time and normal distances of the delta functions $\delta(\rho)=\varepsilon \delta(r)$ and $\delta(\tau)=\varepsilon \delta(t)$.

Now we can see how the small noise assumption has been implemented in our approach. With the choice given by Eqs. (3.1), (3.2) for the orders in $\varepsilon$ of the noise amplitudes, $\varepsilon^{1 / 2} H$ is $O\left(\varepsilon^{2}\right)$ in Eq. (3.17) and $-\varepsilon^{-2} \partial_{\rho} Q_{\rho}$ is $O\left(\varepsilon^{0}\right)$ in Eq. (3.18), i.e. one order higher than the temporal derivatives in these equations. In other words, both noise terms are first order perturbations for the dynamics in their respective inner equations.

At this point, we proceed as in the outer region by expanding the inner fields and parameters in powers of $\varepsilon$

$$
\begin{aligned}
U & =U_{0}+\varepsilon U_{1}+\varepsilon^{2} U_{2}+O\left(\varepsilon^{3}\right), \\
\Phi & =\Phi_{0}+\varepsilon \Phi_{1}+\varepsilon^{2} \Phi_{2}+O\left(\varepsilon^{3}\right), \\
\kappa_{i} & =\kappa_{i 0}+\varepsilon \kappa_{i 1}+O\left(\varepsilon^{2}\right), i=1,2 \\
\Pi & =\Pi_{0}+\varepsilon \Pi_{1}+O\left(\varepsilon^{2}\right), \\
v & =v_{0}+\varepsilon v_{1}+O\left(\varepsilon^{2}\right),
\end{aligned}
$$

and inserting the expansions into the inner equations Eqs. (3.17), (3.18). The inner solutions will be obtained by matching with the outer solutions for $\rho \rightarrow \pm \infty$ and $r \rightarrow 0^{ \pm}$, respectively. In the phase field equations, direct matching with the outer $\phi_{i}$ solutions Eq. (3.15) provides the limiting boundary conditions for the $\Phi_{i}$ terms of the inner expansion

$$
\begin{aligned}
& \Phi_{0}(\rho \rightarrow \pm \infty)=\mp 1, \\
& \Phi_{i}(\rho \rightarrow \pm \infty)=0, \text { for } i=1,2 .
\end{aligned}
$$

Similarly, the matching condition for the inner diffusion field requires that, at leading order, the gradients of $U_{0}$ vanish

$$
\lim _{\rho \rightarrow \pm \infty} \partial_{\rho} U_{0}=0
$$

At higher orders, the matching conditions for the diffusive field present some additional difficulties due to the apparition of random terms, and will be discussed in detail in Sec. IIID. 


\section{Zero order}

At leading order ( $\varepsilon^{0}$ for the $\Phi$ equation, $\varepsilon^{-2}$ for the $U$ equation), the inner equations are given by

$$
\begin{aligned}
& \partial_{\rho}^{2} \Phi_{0}-f^{\prime}\left(\Phi_{0}\right)=0 \\
& \partial_{\rho}^{2} U_{0}=0
\end{aligned}
$$

Inserting the double-well potential Eq. (2.3) into Eq. (3.29), we obtain the standard kink solution for the phase-field at zero-order

$$
\Phi_{0}(\rho)=-\tanh \left(\frac{\rho}{\sqrt{2}}\right)
$$

which satisfies the matching condition Eq. (3.26) for $\rho \rightarrow \pm \infty$. Integrating Eq. (3.30) twice over $\rho$, we have

$$
U_{0}(\rho, \mathbf{s}, \tau)=A(\mathbf{s}, \tau)+B(\mathbf{s}, \tau) \rho
$$

where $A$ and $B$ are integration constants. Imposing the matching condition Eq. (3.28), we determine $B(\mathbf{s}, \tau)=0$ and obtain a $\rho$-independent solution for the diffusion field at zero order

$$
U_{0}(\mathbf{s}, \tau)=A(\mathbf{s}, \tau)
$$

\section{First Order}

Using the solutions obtained at zero-order, the first-order inner equations $\left(\varepsilon^{1}\right.$ for the $\Phi$ equation, $\varepsilon^{-1}$ for the $U$ equation) read

$$
\begin{aligned}
\Omega \Phi_{1} & =-\left(v_{0} \alpha+\kappa_{0}\right) \partial_{\rho} \Phi_{0}+\lambda g^{\prime}\left(\Phi_{0}\right) U_{0} \\
\partial_{\rho}^{2} U_{1} & =\frac{d U_{0}}{d \tau}+\frac{v_{0}}{2} \partial_{\rho} h\left(\Phi_{0}\right)
\end{aligned}
$$

where $\Omega$ is the self-adjoint operator $\Omega \equiv \partial_{\rho}^{2}-f^{\prime \prime}\left(\Phi_{0}\right)$ and we have used that $d \Phi_{0} / d \tau=0$ from Eq. (3.31). As described by Almgren [11], an expression for $\Phi_{1}$ can be obtained from Eq. (3.34) by inverting the operator $\Omega$, leading to

$$
\Phi_{1}=\Omega^{-1}\left[-\left(v_{0} \alpha+\kappa_{0}\right) \partial_{\rho} \Phi_{0}+\lambda g^{\prime}\left(\Phi_{0}\right) U_{0}\right]
$$


Since $\Omega$ is an even operator and $\partial_{\rho} \Phi_{0}, g^{\prime}\left(\Phi_{0}\right)$ are even functions of $\rho, \Phi_{1}$ is an even function of $\rho$. Integrating Eq. (3.35) twice over $\rho$, we get

$$
U_{1}=D(\mathbf{s}, \tau)+C(\mathbf{s}, \tau) \rho+\frac{v_{0}}{2} \int_{0}^{\rho} d \rho^{\prime} h\left(\Phi_{0}\right)+\frac{1}{2} \frac{d U_{0}}{d \tau} \rho^{2}
$$

where $D$ and $C$ are integration constants and we have used that $\partial_{\rho} U_{0}=0$ (cf. Eq. (3.33) ).

\section{Second order}

The stochastic terms appear in the inner equations at second order ( $\varepsilon^{2}$ for the $\Phi$ equation, $\varepsilon^{0}$ for the $U$ equation), which are given by

$$
\begin{aligned}
& \Omega \Phi_{2}=-\left(\alpha v_{1}+\kappa_{1}\right) \partial_{\rho} \Phi_{0}-\left(\alpha v_{0}+\kappa_{0}\right) \partial_{\rho} \Phi_{1}+\alpha \frac{d \Phi_{1}}{d \tau} \\
&+\frac{1}{2} f^{\prime \prime \prime}\left(\Phi_{0}\right) \Phi_{1}^{2}+\rho\left(\kappa_{0}^{2}-2 \Pi_{0}\right) \partial_{\rho} \Phi_{0} \\
&+\lambda g^{\prime}\left(\Phi_{0}\right) U_{1}+\lambda g^{\prime \prime}\left(\Phi_{0}\right) \Phi_{1} U_{0}-\varepsilon^{-3 / 2} H \\
& \partial_{\rho}^{2} U_{2}=-\left(v_{0}+\kappa_{0}\right) \partial_{\rho} U_{1}+\frac{d U_{1}}{d \tau}+\frac{v_{1}}{2} \partial_{\rho} h\left(\Phi_{0}\right)+\frac{v_{0}}{2} \partial_{\rho}\left[h^{\prime}\left(\Phi_{0}\right) \Phi_{1}\right] \\
& \quad-\sum_{i=1,2} \partial_{s_{i}}^{2} U_{0}-\frac{1}{2} h^{\prime}\left(\Phi_{0}\right) \frac{d \Phi_{1}}{d \tau}+\frac{1}{\varepsilon^{2}} \partial_{\rho} Q_{\rho} .
\end{aligned}
$$

The first equation Eq. (3.38) will be used in the next section when imposing the second order solvability condition of the problem. Integrating Eq. (3.39) twice over $\rho$, we find

$$
\begin{aligned}
& U_{2}=F(\mathbf{s}, \tau)+E(\mathbf{s}, \tau) \rho-\left(v_{0}+\kappa_{0}\right) \int_{0}^{\rho} d \rho^{\prime} U_{1} \\
& +\int_{0}^{\rho} d \rho^{\prime} \int_{0}^{\rho^{\prime}} d \rho^{\prime \prime} \frac{d U_{1}}{d \tau}-\frac{1}{2} \partial_{s}^{2} U_{0} \rho^{2}+\frac{v_{1}}{2} \int_{0}^{\rho} d \rho^{\prime} h\left(\Phi_{0}\right) \\
& +\frac{v_{0}}{2} \int_{0}^{\rho} d \rho^{\prime} h^{\prime}\left(\Phi_{0}\right) \Phi_{1}+\frac{1}{\varepsilon^{2}} \int_{0}^{\rho} d \rho^{\prime} Q_{\rho}(\rho, s, \tau),
\end{aligned}
$$

where $F$ and $E$ are again $\rho$-independent integration constants.

\section{Solvability conditions}

We impose now the solvability conditions for the inner problem, which at first and second orders are respectively given by

$$
\int_{-\infty}^{\infty}\left(\partial_{\rho} \Phi_{0}\right) \Omega \Phi_{j} d \rho=0, \text { for } j=1,2 .
$$


Inserting Eq. (3.34) into the first order solvability condition, we get

$$
-\left(\alpha v_{0}+\kappa_{0}\right) I_{1}-\lambda I_{2} U_{0}=0
$$

which allows to determine $U_{0}$ as

$$
U_{0}(\mathbf{s}, \tau)=-\frac{\alpha I_{1}}{\lambda I_{2}} v_{0}-\frac{I_{1}}{\lambda I_{2}} \kappa_{0}
$$

where $I_{1}$ and $I_{2}$ are new integral constants given by

$$
\begin{aligned}
& I_{1}=\int_{-\infty}^{\infty} d \rho\left(\partial_{\rho} \Phi_{0}\right)^{2}, \\
& I_{2}=-\int_{-\infty}^{\infty} d \rho g^{\prime}\left(\Phi_{0}\right)\left(\partial_{\rho} \Phi_{0}\right) .
\end{aligned}
$$

Imposing the second order solvability condition Eq. (3.41), and taking into account the

parity of the potentials $f, g, h$ and of the inner solutions $\Phi_{0}, \Phi_{1}$, we determine an expression for the constant $D$ in Eq. (3.37)

$$
D(\mathbf{s}, \tau)=-\left(\alpha v_{1}+\kappa_{1}\right) \frac{I_{1}}{\lambda I_{2}}+v_{0} \frac{I_{3}}{2 I_{2}}+\frac{I_{4}}{2 I_{2}}+\frac{\alpha I_{5}}{\lambda I_{2}}-\varepsilon^{-3 / 2} \frac{Z(\mathbf{s}, \tau)}{\lambda I_{2}},
$$

where $I_{3}, I_{4}$ and $I_{5}$ are defined by

$$
\begin{aligned}
I_{3} & =\int_{-\infty}^{\infty} d \rho\left(\partial_{\rho} \Phi_{0}\right) g^{\prime}\left(\Phi_{0}\right) \int_{0}^{\rho} d \rho^{\prime} h\left(\Phi_{0}\right), \\
I_{4} & =\frac{d U_{0}}{d \tau} \int_{-\infty}^{\infty} d \rho\left(\partial_{\rho} \Phi_{0}\right) g^{\prime}\left(\Phi_{0}\right) \rho^{2}, \\
I_{5} & =\int_{-\infty}^{\infty} d \rho\left(\partial_{\rho} \Phi_{0}\right) \frac{d \Phi_{1}}{d \tau},
\end{aligned}
$$

and $Z$ is a stochastic term given by

$$
Z(\mathbf{s}, \tau)=\int_{-\infty}^{\infty} d \rho\left(\partial_{\rho} \Phi_{0}\right) H(\rho, s, \tau),
$$

whose statistical properties are given by

$$
\left\langle Z(\mathbf{s}, \tau) Z\left(\mathbf{s}^{\prime}, \tau^{\prime}\right)\right\rangle=2 I_{1} \sigma_{\phi}^{2} \delta\left(\mathbf{s}-\mathbf{s}^{\prime}\right) \delta\left(\tau-\tau^{\prime}\right)
$$

\section{Matching of fluctuating fields}

At this point, we continue by imposing the remaining asymptotic matching conditions of the problem. However, the matching of the diffusion field presents some subtleties due to its 
fluctuating character. The main problem is that, at second order in $\varepsilon$, the $U$ field fluctuates in the normal direction (cf. Eq. (3.40) ), and hence cannot be written as a simple asymptotic expansion for $\rho \rightarrow \pm \infty$, preventing the matching with the outer field. This difficulty can be overcome by introducing an auxiliary matching function defined in both regions as

$$
\begin{aligned}
\chi(r, \mathbf{s}, t) & =u(r, \mathbf{s}, t)-\int_{0}^{r} d r^{\prime} q_{r}\left(r^{\prime}, \mathbf{s}^{\prime}, t\right), \\
X(\rho, \mathbf{s}, \tau) & =U(\rho, \mathbf{s}, \tau)-\int_{0}^{\rho} d \rho^{\prime} Q_{\rho}\left(\rho^{\prime}, \mathbf{s}, \tau\right) .
\end{aligned}
$$

In view of Eq. (3.40), it is easy to see that the inner auxiliary function $X$ introduced in Eq. (3.53) is smooth up to order $\varepsilon^{2}$ in the matching region $r_{M}$. Explicitely, if $X$ is asymptotically expanded for $\rho \rightarrow \pm \infty$ as

$$
X \sim T+S \rho+R \rho^{2}+O\left(\rho^{3}\right)
$$

and the outer matching function $\chi$ is expanded in Taylor around $r=0^{ \pm}$by

$$
\chi \approx \chi\left(0^{ \pm}\right)+\partial_{r} \chi\left(0^{ \pm}\right) \cdot r+\frac{1}{2} \partial_{r}^{2} \chi\left(0^{ \pm}\right) \cdot r^{2}+O\left(r^{3}\right)
$$

the inner and outer terms can be matched at $r_{M} \sim \varepsilon^{1 / 2}$ in the limit $\varepsilon \rightarrow 0$ to obtain the matching relations

$$
\begin{aligned}
T & =\chi\left(0^{ \pm}\right), \\
S & =\varepsilon \partial_{r} \chi\left(0^{ \pm}\right), \\
R & =\frac{\varepsilon^{2}}{2} \partial_{r}^{2} \chi\left(0^{ \pm}\right) .
\end{aligned}
$$

The last step is to expand the previous Equations (3.56), (3.57) and (3.58) in powers of $\varepsilon$ to complete the matching at each order in $\varepsilon$.

\section{First Order}

At first order in $\varepsilon$, the inner field $U_{1}$ given by Eq. (3.37) can be asymptotically expanded for $\rho \rightarrow \pm \infty$ as

$$
U_{1} \sim \frac{1}{2} \frac{d U_{0}}{d \tau} \rho^{2}+\left[C \mp \frac{v_{0}}{2}\right] \rho+D+\frac{v_{0}}{2} J_{1}^{ \pm},
$$

where $D$ is given by Eq. (3.46) and

$$
J_{1}^{ \pm}=\int_{0}^{ \pm \infty} d \rho\left[h\left(\Phi_{0}(\rho)\right) \pm 1\right],
$$


where we have used that $h$ satisfies $h( \pm 1)= \pm 1$ and the far field condition Eq. (3.26). Since $h\left(\Phi_{0}\right)$ is an odd function of $\rho$, we have

$$
J_{1}^{+}=J_{1}^{-} \equiv J_{1}
$$

\section{Second Order}

Similarly, the second order inner solution for the diffusive field Eq. (3.40) can be expanded asymptotically for $\rho \rightarrow \pm \infty$ as

$$
\begin{aligned}
& U_{2} \sim F+E \rho-\left(v_{0}+\kappa_{0}\right) \int_{0}^{\rho} d \rho^{\prime} \frac{d U_{1}}{d \tau}+\int_{0}^{\rho} d \rho^{\prime} \int_{0}^{\rho^{\prime}} d \rho^{\prime \prime} \frac{d U_{1}}{d \tau} \\
& -\frac{1}{2} \partial_{s}^{2} U_{0} \rho^{2}-\int_{0}^{\rho} d \rho^{\prime} \int_{0}^{\rho^{\prime}} d \rho^{\prime \prime} h^{\prime}\left(\Phi_{0}\right) \frac{d \Phi_{1}}{d \tau} \\
& +\frac{v_{0}}{2} J_{2}^{ \pm}+\frac{v_{1}}{2} J_{1} \mp \frac{v_{1}}{2} \rho+\frac{1}{\varepsilon^{2}} \int_{0}^{\rho} d \rho^{\prime} Q_{\rho}(\rho, \mathbf{s}, t),
\end{aligned}
$$

where

$$
J_{2}^{ \pm}=\int_{0}^{\rho} d \rho^{\prime} h^{\prime}\left(\Phi_{0}\right) \Phi_{1}
$$

and we have used the far field conditions Eqs. (3.26) and (3.27). Inserting the expressions Eqs. (3.43), (3.59) and (3.62) into the right hand side of Eq. (3.53), we can determine the parameters $R, S$ and $T$ in the far field expansion of the matching function $X$ (cf. Eq. (3.54) ) and perform the matching with the outer function $\chi$ (cf. Eq. (3.55)).

Imposing the third matching condition Eq. (3.58) at first order in $\varepsilon$, we determine that

$$
\frac{d U_{0}}{d \tau}=0
$$

which, using the relation Eq. (3.36), brings to

$$
\frac{d \Phi_{1}}{d \tau}=0
$$

and therefore the integral constants $I_{4}$ and $I_{5}$ defined in Eqs. (3.48), (3.49) vanish

$$
I_{4}=I_{5}=0
$$

From the two first orders of Eq. (3.56), we get an expression for the outer diffusive field at the interface valid up to first order

$$
u\left(0^{ \pm}\right)=-\frac{I_{1}}{\lambda I_{2}}(\alpha v+\kappa)+\frac{\varepsilon v_{0}}{2}\left(\frac{I_{3}}{I_{2}}+J_{1}\right)-\frac{z(\mathbf{s}, t)}{\lambda I_{2}}+O\left(\varepsilon^{2}\right)
$$


where $z(\mathbf{s}, t)=Z(\mathbf{s}, \tau) \varepsilon^{-1 / 2}$ is a stochastic term whose statistical properties can be determined from Eq. (3.51) and are given by

$$
\left\langle z(\mathbf{s}, t) z\left(\mathbf{s}^{\prime}, t^{\prime}\right)\right\rangle=2 I_{1} \sigma_{\phi}^{2} \delta\left(\mathbf{s}-\mathbf{s}^{\prime}\right) \delta\left(t-t^{\prime}\right) .
$$

Note that the projected interfacial noise term has neither in Eq. (3.67) nor in Eq. (3.68) any explicit dependence in $\varepsilon$, which is a direct consequence of the $\varepsilon^{3 / 2}$ factor introduced in the noise term of Eq. (2.1). Indeed, this is the reason why such factor was introduced in the formulation of the model.

The calculation is completed by imposing the matching condition Eq. (3.57) up to second order, which can be written as

$$
v_{0}+\varepsilon v_{1}=\left.\partial_{r} \chi\right|_{+} ^{-}+O\left(\varepsilon^{2}\right) .
$$

Inserting Eq. (3.52) into Eq. (3.69), we get a heat/mass conservation equation valid up to first order in $\varepsilon$,

$$
v=v_{0}+\varepsilon v_{1}=\left[\partial_{r} u\right]_{+}^{-}-\left[q_{r}\right]_{+}^{-}+O\left(\varepsilon^{2}\right),
$$

where $q_{r}$ accounts for a normal stochastic current across the interface. This term, although being of order $\varepsilon^{2}$, has not been neglected in Eq. (3.70) in order to not break mass conservation in the stochastic diffusion equation Eq. (3.16), which is valid up to second order.

This last equation completes the sharp-interface projection of the stochastic phase-field model of Eqs. (2.1)-(2.5). This projection constitutes the main result of this paper, and is given by the diffusion equation Eq. (3.16), with the noise of Eq. (2.5), supplemented with two moving boundary conditions at the interface: the conservation condition Eq. (3.70), and the Gibbs-Thomson Eq. (3.67), where a new projected interfacial noise appears with correlation given by Eq. (3.68). Note that the projected boundary conditions at the interface Eqs. (3.70), (3.67) are obtained at the order immediately lower than the order at which the asymptotic expansion is performed.

While the general lines of the calculation follow the standard sharp-interface asymptotics, we have included the fluctuation terms during all the procedure, which have been projected in the weak noise limit. This calculation is thus similar to the front dynamics projection performed in Ref. [24]. Indeed, the projected interfacial noise appearing in Eq. (3.67) is the analogous counterpart of the noise term of the projected eikonal front equation of Ref. [24]. 


\section{INTERNAL FLUCTUATIONS IN A GENERIC PHASE FIELD MODEL}

Thus far, the noises considered in this work are intended to account for both external and internal sources of fluctuations. Nevertheless, it is worth pointing out that the resulting stochastic sharp-interface equations are similar to those postulated in the Langevin formulation of solidification due to Karma [13, 14] (see also Ref. [25]), which was constructed to follow equilibrium statistics. This offers the possibility of using the results above to provide generic (not necessarily variational) phase-field models with the correct equilibrium fluctuations. To illustrate this, let us consider the Langevin sharp-interface equations [13, 14]

$$
\begin{aligned}
\partial_{t} u_{S I} & =\nabla^{2} u_{S I}-\nabla \cdot \mathbf{q}^{S I}(\mathbf{r}, t), \\
v_{S I} & =\left[\partial_{r} u_{S I}\right]_{+}^{-}-\left[q_{r}^{S I}\right]_{+}^{-}, \\
u_{S I}(0) & =-d_{0} \kappa-\beta v+\theta(\mathbf{r}, t),
\end{aligned}
$$

where $\mathbf{q}^{S I}$ and $\theta$ are fluctuating terms with correlations given by

$$
\begin{aligned}
\left\langle q_{i}^{S I}(\mathbf{r}, t) q_{j}^{S I}\left(\mathbf{r}^{\prime}, t^{\prime}\right)\right\rangle & =\frac{2 K_{B} T_{M}^{2} c}{L^{2} l^{d}} \delta_{i j} \delta\left(\mathbf{r}-\mathbf{r}^{\prime}\right) \delta\left(t-t^{\prime}\right), \\
\left\langle\theta(\mathbf{s}, t) \theta\left(\mathbf{s}^{\prime}, t^{\prime}\right)\right\rangle & =\frac{2 K_{B} T_{M}^{2} c \beta}{L^{2} l^{d}} \delta\left(\mathbf{s}-\mathbf{s}^{\prime}\right) \delta\left(t-t^{\prime}\right) .
\end{aligned}
$$

The Gibbs-Thompson equation Eq. (4.3) can be compared with Eq. (3.67) and the diffusion equation Eq. (4.1) with Eq. (3.16). This comparison enables the determination of the phasefield parameters in terms of physical and substance parameters, which are given by the equations

$$
\begin{aligned}
\lambda & =\frac{I_{1}}{I_{2} d_{0}}, \\
\alpha & =\frac{\beta}{d_{0}}, \\
\sigma_{u}^{2} & =\frac{K_{B} T_{M}^{2} c}{L^{2} l^{d}}, \\
\sigma_{\phi}^{2} & =\frac{I_{1} K_{B} T_{M}^{2} c \beta}{d_{0}^{2} L^{2} l^{d}} .
\end{aligned}
$$

In the last relations, the two first equations Eqs. (4.6), (4.7) are the usual expressions determined by the standard asymptotic procedure, whereas Eq. (4.8) reflects the identification between the conserved stochastic currents of both phase field model and sharp interface projection. In this sense, a major result of our approach has been the derivation of an expression for the noise strength of the phase-field, that is Eq. (4.9), from the above calculations. 
With this election of the model parameters, the phase-field simulations will present the correct equilibrium statistics in the limit of small interface thickness $\varepsilon \rightarrow 0$. Therefore, the non-variational phase-field formulation of Eqs. (2.1), (2.2) can be used to quantitatively account for thermodynamical fluctuations in solidification processes.

\section{TEST OF THE APPROACH}

In order to test the validity of our approach, we have performed 2D phase-field simulations to obtain the power spectrum of the interfacial fluctuations of a solid-liquid stationary flat interface. Introducing the Fourier transform of the interface position $\xi(\mathbf{r}, t)$ as $\xi_{k}(t)=$ $\int d k \xi(\mathbf{r}, t) e^{-i \mathbf{k r}}$, the power spectrum of a stationary planar front in scaled variables is given by

$$
S(k)=\left\langle\xi_{k} \xi_{-k}\right\rangle=\int \frac{d k^{\prime}}{2 \pi}\left\langle\xi_{k} \xi_{k^{\prime}}\right\rangle=\frac{K_{B} T_{M}}{\gamma} \frac{1}{k^{2}},
$$

where $\gamma=l^{d} L^{2} d_{0} / T_{M} c$ is the scaled interfacial surface energy. In the simulations, space and times have been scaled using length and time scales of $l=10^{-8} \mathrm{~m}$ and $\nu=9 \times 10^{-10} \mathrm{~s}$, respectively. The functions $h, g$ have been chosen to be $h(\phi)=\phi$ and $g^{\prime}(\phi)=\left(1-\phi^{2}\right)^{2}$ so that the model does not have a variational structure. The substance parameters used in the simulations correspond to the values of the pure $\mathrm{SCN}$ in the $3 \mathrm{D}$ case, and are given by $d_{0}=0.2817, \beta=3.0331$ [26, 27] and $\sigma_{u}^{2}=0.001432$. For this choice, and using Eqs. (4.6])(4.9), the phase-field parameters take the values $\lambda=3.13, \alpha=10.76$ and $\sigma_{\phi}^{2}=0.05158$. The interface thickness has been taken to be $\varepsilon=0.3$.

The simulations have been implemented with a finite differences scheme on a $50 \times 512$ lattice with $\Delta x=\Delta y=0.2$ and $\Delta t=0.005$. We have used the initial conditions $\phi(x, y, 0)=-\tanh (x / \varepsilon \sqrt{2}), u(x, y, 0)=0$. Non-flux and periodic boundary conditions have been imposed in the $x$ and $y$ directions respectively.

The numerical implementation of the stochastic terms has been carried out by generating Gaussian-distributed random numbers at each of the lattice sites. The correlations of these numbers can be determined by discretizing the time and spatial delta functions in Eqs. (2.4), (2.5]) by substituting $\delta\left(x-x^{\prime}\right) \rightarrow \delta_{i i^{\prime}} / \Delta x$ and $\delta\left(t-t^{\prime}\right) \rightarrow \delta_{n n^{\prime}} / \Delta t$. The divergence of the stochastic current in Eq. (2.2) has been discretized by using a forward differences scheme $\left.\nabla \cdot \mathbf{q}(\mathbf{r}, t)\right|_{i, j}=\left(q_{x}(i+1, j)-q_{x}(i, j)\right) / \Delta x+\left(q_{y}(i, j+1)-q_{y}(i, j)\right) / \Delta y$.

The power spectrum statistics has been obtained as a time average among the last $3 \times 10^{6}$ 


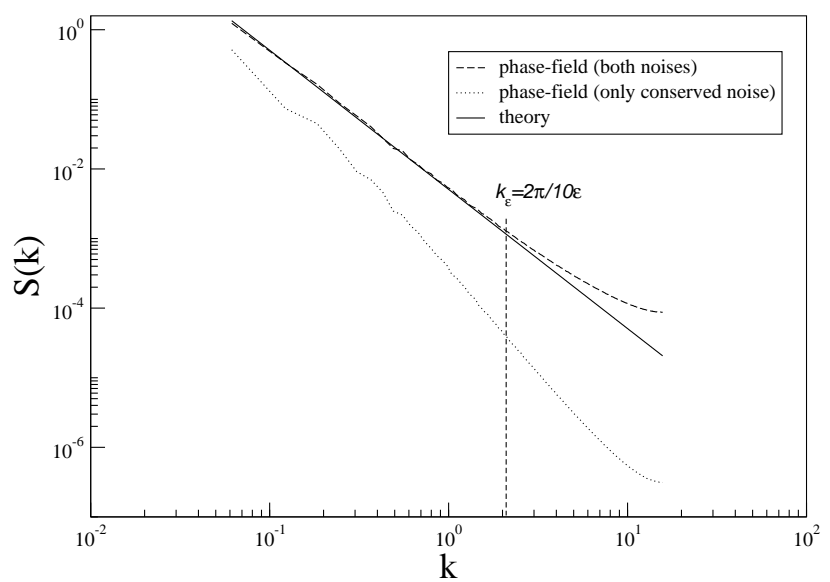

FIG. 1: Comparison between the theoretical power spectrum of the stationary interface and the results from the phase-field simulations.

time steps in a long-term simulation of $3.5 \times 10^{6}$ steps, and is represented by a dashed line in Fig. 1. The solid line in Fig. 11 depicts the theoretical prediction given by Eq. (5.1) and, as it can be seen, an excellent agreement is found between theoretical and numerical results. The vertical dashed line in the figure represents the wavelength associated with the effective thickness of the interface, and determines the expected breakdown of the phase-field description.

\section{DISCUSSION AND CONCLUSIONS}

To summarize, we have obtained an asymptotic projection of the fluctuating phase-field equations (2.1), (2.2) to a sharp interface description. This has been worked out by means of a hybrid asymptotic procedure, combining sharp interface and small noise limits. As a result, the projected equations adopt the form of a moving boundary problem with a conserved stochastic force $\mathbf{q}$ in the equations for the diffusion field Eqs. (3.16) and (3.70), and an interfacial noise $z(\mathbf{s}, t)$ in the Gibbs-Thompson condition Eq. (3.67). Other authors have previously introduced fluctuations in phase-field models [20, 21, 22], but their approaches applied only for the case of variational formulations and were restricted to noises from a thermodynamical origin.

In this context, it has been claimed [20] that the presence of a non-conserved noise such as $\eta$ in the equation for the phase field is not relevant for the dynamics of the phase field 
model, and thus could be omitted in simulations. In order to check the importance of the non-conserved phase field noise, we have carried out a numerical test with the same parameters reported in section $\nabla$ but taking $\sigma_{\phi}=0$. In this case, the power spectrum is plotted as a dotted line in Fig. 1. The clear disagreement with both the theoretical prediction and the simulations of the complete model indicates that the phase field noise is indeed necessary in order to obtain quantitative results. This can be explained by noting that Eq. (3.67) establishes a direct relation between the non-conserved phase-field noise $\eta$ and the interfacial fluctuations appearing in the Gibbs-Thompson equation, usually associated to kinetic attachment effects [13]. Thus, the small significance of the $\eta$ noise reported in Ref. [20] is probably due to the fact that the stationary power spectrum was calculated in the limit of vanishing kinetics $\beta=0$. Therefore, we conclude that, in the presence of kinetic effects, the phase field noise is relevant for a quantitative description of the solidification process.

It is interesting to discuss the scaling of the noise terms as proposed on the one hand in Eqs. (3.1), (3.2) and on the other hand in the $\varepsilon^{3 / 2}$ factor explicitly appearing in the equation for the phase field, Eq. (2.1). As it has already been commented, the assumption of the order relations Eqs. (3.1), (3.2) has permitted to manage a double expansion (sharp interface and small noise) by formally using a single small parameter. The specific powers of $\varepsilon$ appearing in these relations have been chosen for maintaining fluctuations as small perturbations for the dynamics of both inner and outer equations. On the contrary, the multiplicative factor of the noise term of the equation for the phase field, Eq. (2.1), has a different motivation. It is well known that there are problems in the formulation of stochastic field equations when the noise terms are delta-correlated in space. I such cases, some kind of regularization is required. In Ref. 24], for instance, this regularization was provided by the correlation length of the noise, in such a way that the results did depend on that parameter. In the present case, the regularization is provided by the interface width $\varepsilon$. The projection of the bulk noise into the interface gives a fluctuation term that in principle should diverge as $\epsilon$ goes to zero. The $\epsilon^{3 / 2}$ factor of the noise term in Eq. (2.1) exactly cancels out this divergence, and has been introduced in the formulation of the model precisely to make results independent of $\epsilon$, specifically regarding the new interfacial noise term $z$ in Eqs. (3.67), (3.68).

In conclusion, we have proposed an asymptotic procedure to obtain the sharp-interface projection of a generic (not necessarily variational) phase-field model with fluctuations. We 
have tested the validity of our approach by comparing the phase-field results with the theoretical prediction for the interfacial fluctuations in a simple solidification problem. This procedure can be useful both in situations where the fluctuation-dissipation theorem does not hold, such as in presence of external fluctuations, and when the phase-field model has a non-variational nature. An important example of this latter case corresponds to efficient phase-field models for the solidification of alloys [4, 12], which with our method can incorporate internal fluctuations. The availability of these models for quantitative simulations in the fluctuating case appears as a promising step towards the study of complex situations such as the apparition of dendritic sidebranching or the wavelength selection during initial redistribution transients in the directional solidification of alloys [28, 29].

\section{ACKNOWLEDGMENTS}

This work was financially supported by Dirección General de Investigación Científica y Técnica (Spain) (Project BFM2003-07850-C03-02) and Comissionat per a Universitats i Recerca (Spain) (Project 2001/SGR/00221). We also acknowledge computing support from Fundació Catalana per a la Recerca through C4 and CESCA (Spain).

[1] R. González-Cinca, R. Folch, R. Benítez, L. Ramírez-Piscina, J. Casademunt, and A. Hernández-Machado, in Advances in Condensed Matter and Statistical Mechanics, edited by E. Korutcheva and R. Cuerno (Nova Science Publishers, 2004), pp. 203-236, (condmat/0305058).

[2] A. A. Wheeler, W. J. Boettinger, and G. B. McFadden, Phys. Rev. A 45, 7424 (1992).

[3] A. A. Wheeler, W. J. Boettinger, and G. B. McFadden, Phys. Rev. E 47, 1893 (1992).

[4] A. Karma and W. J. Rappel, Phys. Rev. E 57, 4323 (1998).

[5] J. A. Warren, R. Kobayashi, A. E. Lobkovsky, and W. C. Carter, Acta Materialia 51, 6035 (2003).

[6] I. S. Aranson, V. A. Kalatsky, and V. M. Vinokur, Phys. Rev. Lett. 85, 118 (2000).

[7] R. Folch, J. Casademunt, A. Hernández-Machado, and L. Ramírez-Piscina, Phys. Rev. E 60, 1724 (1999). 
[8] T. Biben and C. Misbah, Phys. Rev. E 67, 031908 (2003).

[9] G. Caginalp and P. Fife, SIAM J. Appl. Math. 48, 506 (1988).

[10] K. R. Elder, M. Grant, N. Provatas, and J. M. Kosterlitz, Phys. Rev. E 64, 021604 (2001).

[11] R. F. Almgren, SIAM J. Appl. Math. 59, 2086 (1999).

[12] A. Karma, Phys. Rev. Lett. 87, 115701 (2001).

[13] A. Karma, Phys. Rev. E 48, 3441 (1993).

[14] A. Karma, Phys. Rev. Lett. 70, 3439 (1993).

[15] J. A. Warren and J. S. Langer, Phys. Rev. A 42, 3518 (1990).

[16] J. A. Warren and J. S. Langer, Phys. Rev. A 47, 2702 (1993).

[17] J. Langer, Phys. Rev. A 36, 3350 (1987).

[18] R. Kobayashi, Physica D 63, 410 (1993).

[19] K. R. Elder, F. Drolet, J. M. Kosterlitz, and M. Grant, Phys. Rev. Lett. 72, 677 (1994).

[20] A. Karma and W. J. Rappel, Phys. Rev. E 60, 3614 (1999).

[21] S. G. Pavlik and R. F. Sekerka, Physica A 268, 283 (1999).

[22] S. G. Pavlik and R. F. Sekerka, Physica A 277, 415 (2000).

[23] P. C. Hohenberg and B. I. Halperin, Rev. Mod. Phys. 49, 435 (1977).

[24] A. Rocco, L. Ramírez-Piscina, and J. Casademunt, Phys. Rev. E 65, 056116 (2002).

[25] T. A. Cherepanova, Sov. Phys. Dokl. 21, 109 (1976).

[26] A. Chernov, J. Crystal Growth 264, 499 (2004).

[27] M. Aziz and W. Boettinger, Acta Metall. Mater. 42, 527 (1994), the value of $\beta$ has been determined by assuming a diffusion-limited kinetic attachment $\tilde{\mu}=\tilde{v}^{d} L / R T_{M}^{2}$, where $\tilde{v}^{d}=$ $\tilde{D} / \tilde{d}_{0}$, being $\tilde{D}$ the thermal diffusivity and $\tilde{d}_{0}$ the capillary length, written in physical units.

[28] R. Benítez and L. Ramírez-Piscina, Fluct. Noise Lett. 4, L505 (2004).

[29] R. Benítez and L. Ramírez-Piscina, in preparation. 